\title{
Recent infection by Wolbachia alters microbial communities in wild Laodelphax striatellus populations
}

Xing-Zhi Duan ${ }^{1 \dagger}$, Jing-Tao Sun ${ }^{1 \dagger}$, Lin-Ting Wang ${ }^{1}$, Xiao-Han Shu', Yan Guo ${ }^{1}$, Matsukura Keiichiro ${ }^{2}$, Yu-Xi Zhu', Xiao-Li Bing ${ }^{1}$, Ary A. Hoffmann ${ }^{3}$ and Xiao-Yue Hong ${ }^{1 *}$ (D)

\begin{abstract}
Background: Host-associated microbial communities play an important role in the fitness of insect hosts. However, the factors shaping microbial communities in wild populations, including genetic background, ecological factors, and interactions among microbial species, remain largely unknown.

Results: Here, we surveyed microbial communities of the small brown planthopper (SBPH, Laodelphax striatellus) across 17 geographical populations in China and Japan by using 16S rRNA amplicon sequencing. Using structural equation models (SEM) and Mantel analyses, we show that variation in microbial community structure is likely associated with longitude, annual mean precipitation (Bio12), and mitochondrial DNA variation. However, a Wolbachia infection, which is spreading to northern populations of SBPH, seems to have a relatively greater role than abiotic factors in shaping microbial community structure, leading to sharp decreases in bacterial taxon diversity and abundance in host-associated microbial communities. Comparative RNA-Seq analyses between Wolbachia-infected and -uninfected strains indicate that the Wolbachia do not seem to alter the immune reaction of SBPH, although Wolbachia affected expression of metabolism genes.

Conclusion: Together, our results identify potential factors and interactions among different microbial species in the microbial communities of SBPH, which can have effects on insect physiology, ecology, and evolution.
\end{abstract}

Keywords: Laodelphax striatellus, Microbial community, Wolbachia, Endosymbiont, Microbial interactions

\section{Background}

The fitness of insects can be affected by their interactions with associated microbiomes [1-3]. Fitness traits affected by host microbiomes include development [4], fecundity [5], resistance to natural enemies [6], climate adaptation [7], and synthesis of essential amino acids $[8,9]$. In addition, disturbing an insect's bacterial population can change host fitness [10], such as producing enhanced

\footnotetext{
* Correspondence: xyhong@njau.edu.cn

${ }^{+}$Xing-Zhi Duan and Jing-Tao Sun contributed equally to this work.

'Department of Entomology, Nanjing Agricultural University, Nanjing 210095, Jiangsu, China

Full list of author information is available at the end of the article
}

sensitivity to bacterial pathogens in bees [11] and altering fecundity in mosquitos $[12,13]$.

The microbial communities of hosts are influenced by diverse factors that include diet [14], $\mathrm{pH}$ [15], host [16], life stage [17], temperature and humidity [18], and genetic background [19]. Evidence for effects of genetic background on microbial communities is mostly based on correlations between microbial structure and phylogenetic relationships at the macro-evolutionary level [20, 21], although such correlations might reflect factors like geographic isolation that drive speciation rather than genetic backgrounds per se. Apart from external factors, changes in microbial communities can also be driven by interactions between different microbial 
species [22]. For example, Wolbachia has been shown to compete against pathogens in Drosophila [23] and Aedes [24, 25]. Similarly, Spiroplasma reduces the density of Wolbachia in Drosophila [26] and Asaia impedes the vertical transmission of Wolbachia in Anopheles stephensi mosquitoes [27]. Mechanisms involved in these microbial interactions are often not clear.

To understand factors influencing the microbial distribution within hosts, investigations are needed at the population level when there are likely to be fewer confounding effects than in interspecific comparisons across hosts. Here, we undertake such an investigation on the small brown planthopper (SBPH, Laodelphax striatellus), a notorious agricultural pest that damages rice plants by sucking rice sap and spreading rice stripe virus (RSV) [28]. The SBPH has a strong migratory ability but also shows population genetic differentiation $[29,30]$, providing a suitable model for studying the impact of genetic background on microbiomes. Previous studies of the microbiota of $\mathrm{SBPH}$ have relied on laboratory samples [31-33]. However, stable laboratory rearing conditions are likely to alter the original microbial community structure which might be shaped by their original environmental conditions, with a homogenizing effect on the microbial community [34-36]. Moreover, genetic drift can occur, affecting the genetic background of both the host and the microbial community during rearing, generating potential differences between the microbial communities observed in the lab and the field. Given these concerns, our current study focusses on natural populations. We combine $16 \mathrm{~S}$ rRNA amplicon sequencing with a transcriptome analysis to test factors shaping the microbial community in their host at the population level, and we explore the nature of the interactions between different microbial species.

\section{Methods \\ Sample collection}

SBPH individuals were collected from rice plants at 17 locations in China and Japan during the summers (May to September) of 2010-2018 (Fig. 1, left panel; Additional file 1: Table S1). We haphazardly collected about 60-100 adult female individuals at each location. To avoid sampling siblings, we collected only one SBPH per host plant and selected host plants that were at least $1 \mathrm{~m}$ apart. All samples were preserved in $100 \%$ ethanol and stored at $-20^{\circ} \mathrm{C}$ until DNA extraction.

\section{S rRNA amplicon sequencing}

For each of the 17 locations, three female adults were pooled to provide a biological replicate and three biological replicates were established per location. Total genomic DNA was extracted with a DNeasy blood and tissue kit (Qiagen, Hilden, Germany) according to the manufacturer's protocols. A two-step PCR approach recommended by Illumina [37] was performed to generate amplicon libraries. Briefly, the PCR amplification of the bacterial 16S rRNA genes involved universal primer sets 338F (5'-ACTCCTACGGGAGGCAGCAG-3 $\left.{ }^{\prime}\right)$ and 806R (5'-GGACTACHVGGGTWTCTAAT-3'). The PCR products were purified on a $2 \%$ agarose gel, and extracted with an AxyPrep DNA Gel Extraction Kit (Axygen Biosciences, Union City, CA, USA). The Illumina sequencing adapters and sample-specific barcodes were added to the purified PCR products with a second PCR using the TruePrep Index Kit V3 for Illumina (Vazyme, Nanjing, China). Final PCR products were purified with Hieff NGS DNA selection Beads (YEASEN, Shanghai, China), and equalized and normalized using the dsDNA HS assay kit for Qubit (YEASEN, Shanghai, China). Samples were quantified and pooled in equimolar ratio using a Qubit 4 Fluorometer (Invitrogen, Waltham, MA, USA) and then were submitted to Majorbio Bio-Pharm Technology Company Limited (Shanghai, China) for high-throughput sequencing on an Illumina MiSeq PE300.

After sequencing, raw fastq files were demultiplexed, quality-filtered by Trimmomatic, and merged by FLASH [38] (http://www.cbcb.umd.edu/software/flash). OTUs were clustered with $97 \%$ similarity cutoff using UPARSE [39] (version 7.1, http://drive5.com/uparse/) and sequences were then phylogenetically assigned to taxonomic classifications using an RDP classifier [40] (http:// rdp.cme.msu.edu/). To normalize sequencing depth, the samples were rarefied to 34135 sequences (the lowest coverage sample) to ensure a random subset of OTUs for all samples.

\section{Mitochondrial $\mathrm{COI}$ gene PCR}

In $\mathrm{SPBH}$, no significant differentiation among populations exists for nuclear genes but mitochondrial genes that are passed down from mother are differentiated [29]. To determine the degree of genetic differentiation, 20 to 46 female adults were haphazardly selected from each population (Fig. 1, left panel) for mitochondrial COI gene amplifications and sequencing according to Sun et al. [29]. The PCR products were sent to Tsingke Biological Technology Company (China) for sequencing.

\section{Diagnostic PCR}

To measure infection frequencies of Wolbachia, an additional eight to 46 female adults were haphazardly selected from each population. The specific primers [41] are listed in Additional file 1: Table S2. DNA extraction and PCR were done as described above. Positive controls (known sample with Wolbachia) and blank controls were also run. PCR products of $599 \mathrm{bp}$ size were run on 


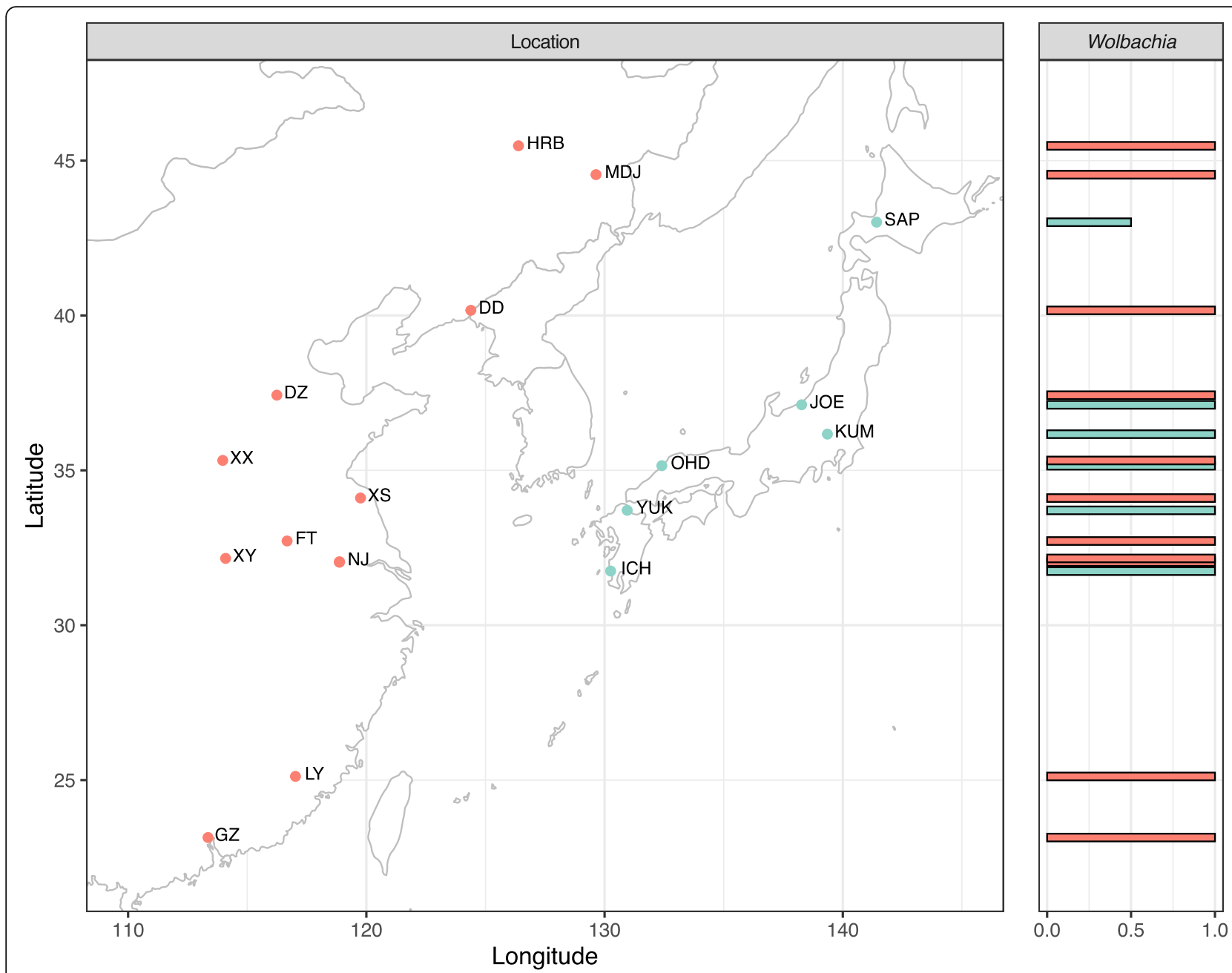

Fig. 1 Sampling localities (left) and infection frequencies (right) of Wolbachia in natural populations of SBPH across 17 locations. The numbers in the location map indicate the numbers of SBPH detected. Positions of the infection frequency bars correspond to the latitude of the population. The locations and dates of collection are given in Additional file 1: Table S1

$1.0 \%$ agarose gels stained with ethidium bromide at 150 volts and visualized by GenoSens 1860 (Clinx, Shanghai, China). The number of samples showing bright DNA bands compared with the DL 2000 DNA mark (Tsingke, China) was used to calculate the infection rate.

\section{Transcriptome analyses}

To investigate the effects of Wolbachia infection on the SBPH transcriptome, we compared Wolbachia-free and Wolbachia-infected SBPH strains. The uninfected strain was obtained by treating the infected strain with tetracycline for 10 generations according to the method of Guo et al. [42]. Briefly, approximately 30 abdomens of SBPH as a biological replicate were dissected from 3-day-adults of both Wolbachia-infected and Wolbachia-free females. The female abdomens contain a large quantity of fat body and blood cells which are the basis of innate immunity. Total RNA was extracted from three biological replicates using TRIzol Reagent (Invitrogen, CA, USA) according to the manufacturer's instructions. RNA purity was measured with a NanoPhotometer ${ }^{\bullet}$ spectrophotometer (IMPLEN, CA, USA). RNA concentration was measured with a Qubit $^{\circ}$ RNA Assay Kit in a Qubit $^{\circ}$ 2.0 Fluorometer (Life Technologies, CA, USA). Finally, RNA was pooled for Illumina MiSeq sequencing (BGI, Wuhan, China) according to a standard protocol [43].

The sequencing generated $6.6 \mathrm{~Gb}$ per biological replicate. Clean reads were obtained by removing reads with adaptors, poly-N, and having a low quality. Gene expression levels were estimated by RSEM software package [44] (http://deweylab. biostat.wisc.edu/rsem). Immune-related genes of SBPH were obtained from Zhu et al. [45], which were generated by alignments with immune genes of D. melanogaster, A. gambiae, Aedes aegypti, and Culex quinquefasciatus by using BLASTX [46]. In addition, sequences were annotated to the $\mathrm{KO}$ database with the KEGG Automatic Annotation Server. 


\section{Statistical analyses}

Bray-Curtis dissimilarity metrics among all samples were constructed using beta_diversity.py in QIIME [47] (http://qiime.sourceforge.net/) and were visualized with a principal coordinate analysis (PCoA). The difference of microbial communities among the populations was calculated by ADONIS. The population genetic differentiation value $\left(F_{\mathrm{ST}}\right)$ was calculated in Arlequin 3.5 [48]. The annual mean temperatures (Bio1) and the annual mean precipitation (Bio12) of the 17 locations were obtained from DIVA-GIS 7.5.0 [49] (https://www.diva-gis. org), which is a geographic information system for the analysis of species distribution data. A structural equation model (SEM) [50] was used to estimate the relative contributions of $F_{\mathrm{ST}}$, Bio1, Bio12, latitude, and longitude (Additional file 1: Table S3; Table S4; Table S1) on microbial community structure with communities based on Bray-Curtis dissimilarity metrics. The SEM tests were performed in the R "SEM" package (https://cran.r-project.org/web/packages/sem/index.html), and the path diagram for the SEM tests is shown in Fig. 4. As nonnormal distribution of variables may compromise SEM analyses results, we also undertook Mantel tests using the Spearman method with 1000 permutations to determine the associations between microbial community structure variation and the five aforementioned factors.

The relative abundance of a given phylogenetic group was estimated by examining the number of reads of that group for each population. In order to analyze the evenness and richness of the microbial community, we calculated several $\alpha$ diversity indexes including the Sods, Shannon, Simpson, Ace, Chao, and Coverage indexes. Spearman's rank correlations were calculated between the proportion of Wolbachia and the $\alpha$ diversity indexes (Shannon indexes and Simpson indexes) of the populations. The significance of differences in read proportions of bacterial 16S rRNA genes at the genus level was assessed by Mann-Whitney $U$ tests. The significance of differences in $\alpha$ diversity indexes between Wolbachia-infected and -uninfected populations was calculated by a $t$ test. All statistical analyses were carried out in R 3.5.2 [51].

\section{Probabilistic features recognition for the OTU distribution} Components of collective ecological and biological systems presented an obvious probabilistic similarity in their aggregation, in which only several species made up a relatively high share of the whole sample, while most species accounted for much less. By looking into our datasets, we noted that the abundance data of OTUs explicitly met this property. Therefore, the power-law function that satisfied the mathematical characterization of such distribution behavior was considered as an appropriate function to recognize the probability distribution features of OTUs. Given the type of power-law function, the abundance had the probability density function (pdf):

$$
p(x)=a x^{-\varepsilon}, x>x^{\prime}
$$

where $x^{\prime}$ was the threshold that ensured a robust fitting for the power-law distribution. We probabilistically characterized the distribution of abundance of OTUs by calculating the exceedance probability distribution function [52] that was given by:

$$
P(X \geq x)=x^{1-\varepsilon} f \cdot\left(\frac{x}{x^{\prime}}\right)
$$

where $\varepsilon$ was the scale exponent of power-law distribution underlying the statistical patterns of data considered. This scale factor implied the property of mean and variance of data: when $\varepsilon \leq 2$, the mean and variance were both infinite; when $2<\varepsilon<3$, the mean existed, while the variance was still infinite; and when $\varepsilon \geq 3$, both mean and variance existed. Additionally, $f\left(\frac{x}{x^{\prime}}\right)$ was introduced to give a general formulation for the homogeneity function. The probabilistic features for the OTU distribution for each population were given in Fig. 2 .

To assess the microbial community variations between populations in terms of probabilistic distributions of OTUs, we calculated the Kullback-Leibler divergence (KL divergence) by using the $\mathrm{R}$ package "LaplacesDemon" (https://cran.rproject.org/web/packages/LaplacesDem on/index.html). Probability density functions of OTUs used as the arguments for KL divergence calculation function were computed by using the $\mathrm{R}$ package "histogram" (https://cran.r-project.org/web/packages/ histogram/index.html). The KL divergence was used as a surrogate index of microbial community structure and was also used for SEM and Mantel tests to analyze the relationships between microbial community structure and five putative predictor variables as mentioned above.

\section{Results \\ Microbial diversity and environmental factors in the absence of Wolbachia}

Based on the infection frequencies of Wolbachia, only the SAP population was found to have Wolbachia-uninfected individuals. And a notable difference in microbial community structure was found between SAP and the remaining populations as showed by the probabilistic features of the OTU distribution (Fig. 2). To eliminate the potential influence of Wolbachia on pooled samples, the SAP population was excluded for testing the impact of other factors on the microbial community. Among the 48 samples from the remaining $16 \mathrm{SBPH}$ populations, the RDP classifier identified a total of 314 OTUs (Additional file 2: Table S5). Wolbachia were the most abundant bacteria, accounting for $87.9 \%$ of the $16 \mathrm{~S}$ 


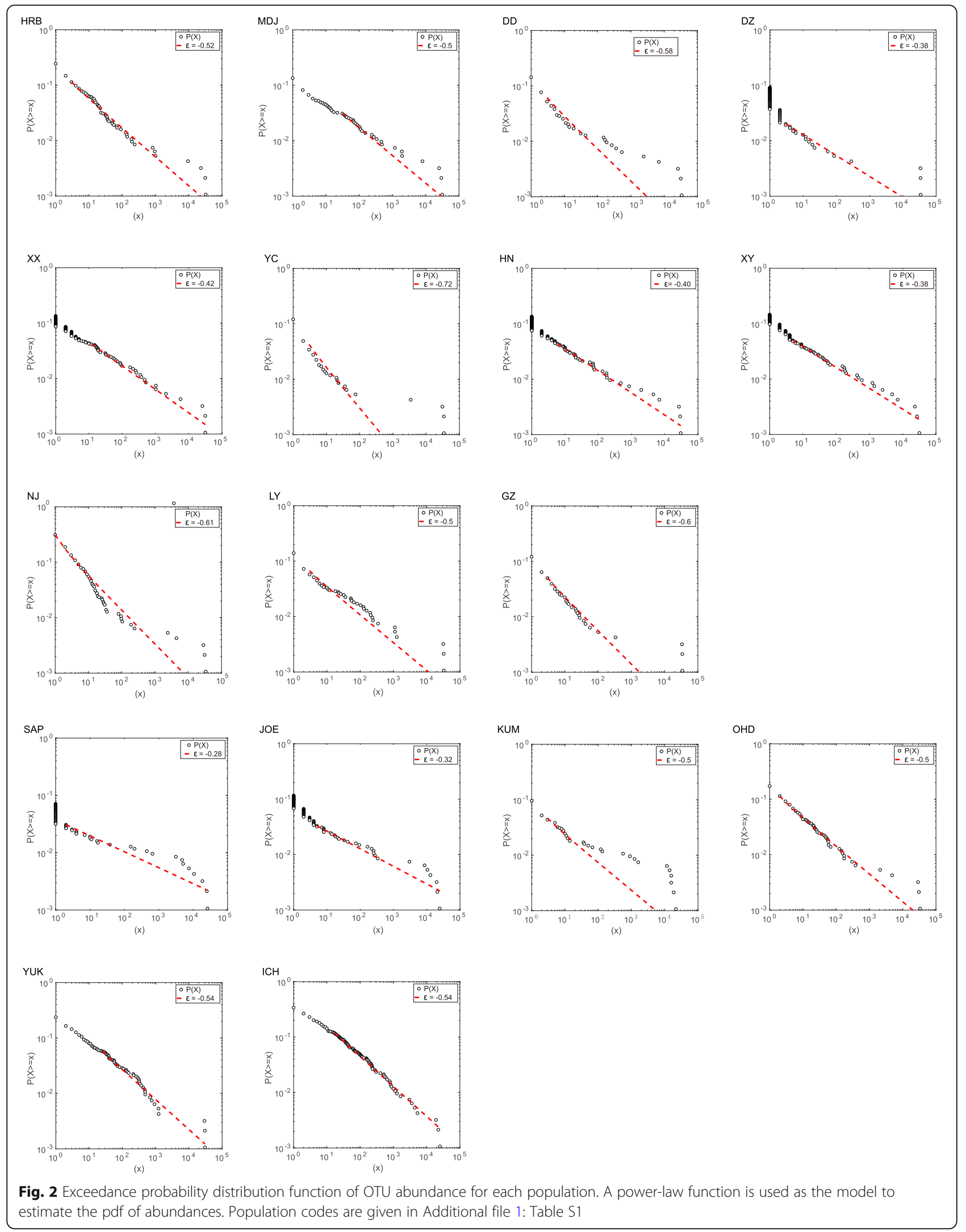


rRNA gene reads in the Chinese populations and $66.4 \%$ of the 16S rRNA gene reads in the Japanese populations (Additional file 1: Table S6). Other prominent genera included Spiroplasma (3.55\%), Asaia (2.47\%), Pantoea (1.04\%), and Herbaspirillum (1.03\%) in the Chinese populations and Diplorickettsia (10.9\%), Asaia (5.56\%), Spiroplasma (5.00\%) and Pantoea (2.08\%) in the Japanese populations. Genera other than Wolbachia were enriched in the Japanese populations compared with the Chinese populations. These results suggest that the structures of SBPH-associated bacteria were different between the two countries.

We found significant differences in microbial communities among the 16 populations (Fig. 3b) based on Bray-Curtis dissimilarity (ADONIS, $r=0.428, p=0.001$ ) and considerable variations based on probabilistic features of the OTU distribution (Fig. 2). To understand whether and to what extent host genetic and environmental factors contributed to variation in microbial communities across the populations, structural equation model (SEM) was used to resolve the relationships between microbial community structure and five putative predictor variables $F_{\mathrm{ST}}$, Bio1, Bio12, latitude, and longitude. The results showed that differences in the microbial community structure characterized by Bray-Curtis dissimilarity could be significantly explained by longitude and annual mean precipitation (Bio12), suggesting geographical location and precipitation help shape the microbial community structure in SBPH (Fig. 4a; Additional file 1: Table S7). However, no significant association between $\mathrm{KL}$ divergence based on the probability densities of OTUs and any geographic or environmental factors was detected by SEM and the Mantel tests (Fig 4b; Table 1; Additional file 1: Table S7).

Pairwise $F_{\mathrm{ST}}$ values computed from the mitochondrial COI gene $(887 \mathrm{bp})$ for the 16 populations showed that
64 of the 120 pairwise population comparisons were significantly different (Additional file 1: Table S3). The SEM analyses also showed significant effects of $F_{\mathrm{ST}}$ on the microbial Bray-Curtis dissimilarity (Fig. 4a; Additional file 1: Table S7), suggesting that mtDNA background correlated with similarity in the microbial community structure among populations. In addition, latitude was found to be associated with $F_{\mathrm{ST}}$. In line with the SEM analyses, Mantel tests showed that longitude and Bio12 significantly correlated with microbial Bray-Curtis dissimilarity (Table 1). However, although an effect of $F_{\mathrm{ST}}$ was detected in the SEM, the correlation from the Mantel test was not significant $(r=0.162, p=0.153)$. This may reflect the lower sensitivity of the Spearman method and reduced effect of genetic background relative to the other two factors. For the analyses based on KL divergence, the SEM analyses showed that KL divergence significantly correlated with $F_{\mathrm{ST}}$ values (Fig. 4b; Additional file 1: Table S7) but the Mantel test was marginally non-significant (Table 1 ).

\section{Effects of Wolbachia on population variation in microbial communities}

The $16 \mathrm{~S}$ rRNA gene data revealed microbial community structure across populations at the genus level (Fig. 3a). The proportions of Wolbachia reads in the high latitude populations of Japan (KUM, JOE, and SAP) were low. To test whether the spread of Wolbachia might affect microbial community structure, diagnostic PCR was conducted to assess the frequency of Wolbachia across the 17 populations (Fig. 1, right panel). The results showed that the infection rate of Wolbachia in SAP was $50 \%$ while it was $100 \%$ in the other populations. While these results are similar to previous findings in $\mathrm{SBPH}$
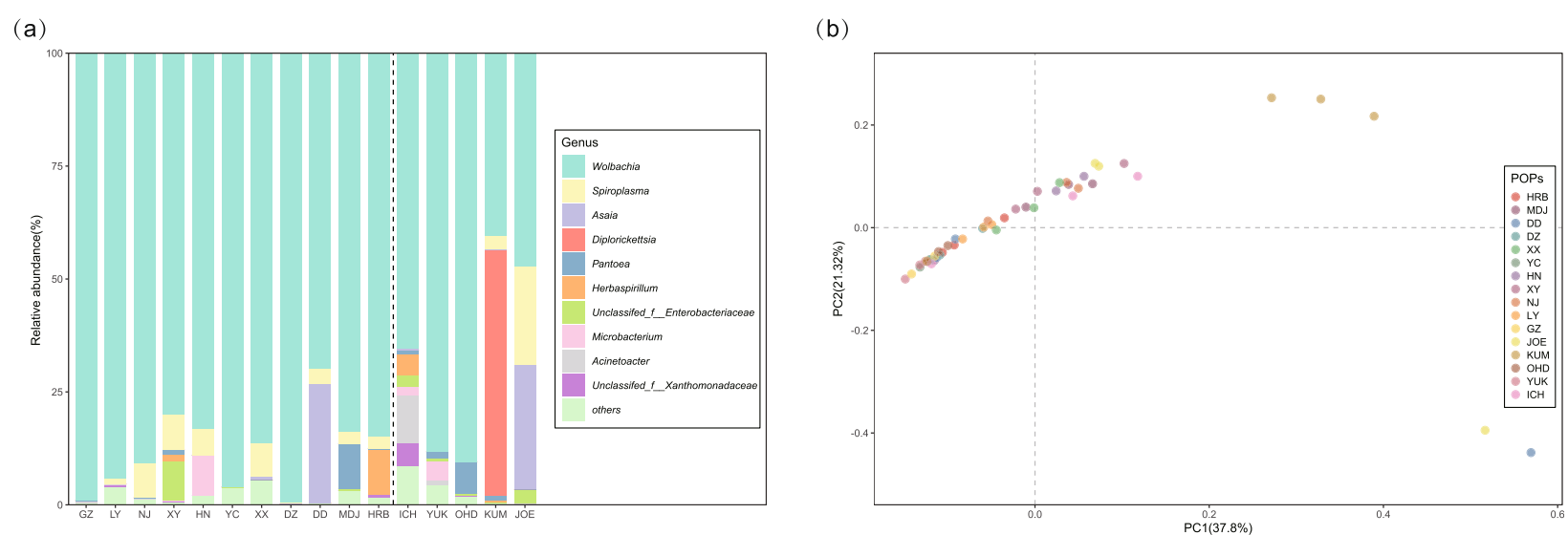

Fig. 3 The abundance and distance of microbial communities of SBPH across 16 populations. a Relative abundance of bacterial $16 \mathrm{~S}$ rRNA genes at the genus level. Dashed line separates the Chinese and Japanese microbial community abundance. Blocks of populations were arranged by origin sites (south to north). Other genera ("others") account for < 5\% of the classified sequences. b Principal coordinate analysis (PCoA) of SBPH samples collected from different locations. PCoA was generated by the Bray-Curtis dissimilarity method 
(a)

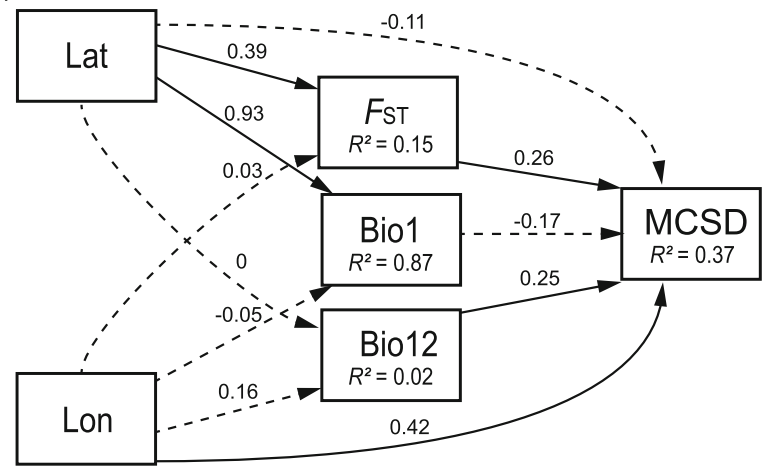

(b)

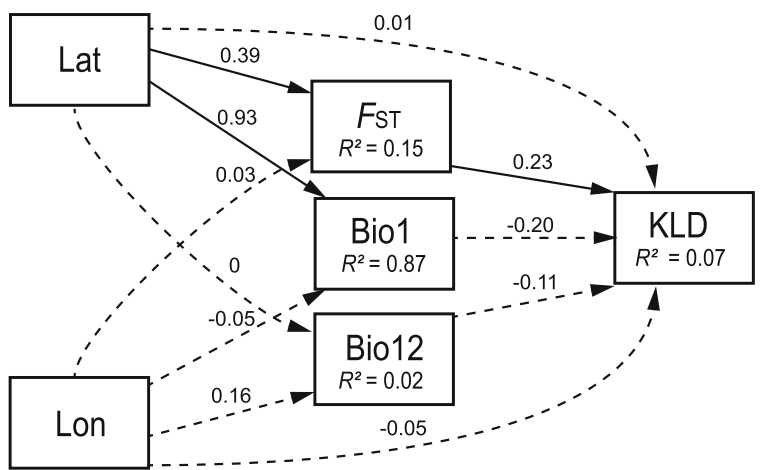

Fig. 4 Path diagram for the structural equation model (SEM) for a environmental/genetic factors and microbial Bray-Curtis dissimilarity, and $\mathbf{b}$ environmental/genetic factors and KL divergence in natural populations of SBPH. Statistically significant positive paths are indicated by solid arrows. Statistically significant negative paths are indicated by dashed arrows. The $R^{2}$ values in each box indicate the amount of variation in that variable explained by the input arrows. Numbers next to arrows are unstandardized slopes. Lat, Latitude; Lon, Longitude; MCSD, microbial BrayCurtis dissimilarity; KLD, KL divergence

showing a relatively higher Wolbachia incidence in China [53], the frequency of Wolbachia infection observed in the present study was higher than it was in the previous studies, especially in Japan [54]. This showed that Wolbachia has increased in recent decades.

The correlations between the $\alpha$ diversity indexes (Shannon and Simpson indexes) $[55,56]$ and the proportion of Wolbachia in all samples were examined by Spearman's rank correlation analyses (Fig. 5a, b). The results revealed that the proportions of Wolbachia were significantly correlated with the Shannon $(r=-0.940, P<0.001)$ and Simpson $(r=0.979, P<0.001)$ indexes, suggesting that the presence of Wolbachia in SPBH decreased the richness and evenness of microbial communities.

\section{Wolbachia infection and the relative abundance of bacterial taxa in SPBH}

To further test the impact of Wolbachia infection on the microbial communities, 10 female adults infected with Wolbachia and 9 female adults uninfected with
Wolbachia, both from the SAP population, were used to compare the microbial communities by $16 \mathrm{~S}$ rRNA amplicon sequencing. After the samples were rarefied to 39,872 sequences (the lowest coverage sample), 1985 OTUs were obtained between the two groups (Additional file 2: Table S8). Wolbachia predominated in the microbial communities of Wolbachia-infected females (Fig. 6a). The relative abundances of 154 genera in the Wolbachia-infected adults were significantly reduced (Additional file 2: Table S9). PCoA analysis based on Bray-Curtis dissimilarity (Fig. 6b) clearly separated the Wolbachia-infected individuals from the Wolbachia-uninfected individuals, indicating that the microbial community structures of the two groups were significantly different. Compared to the Wolbachia-infected group, the Wolbachia-free group possessed high microbial diversity as suggested by the Sobs, Shannon, Simpson, Chao. and Ace indexes (Table 2: Welch two-sample $t$ test: $p=0.003$ for Sobs, $p<0.001$ for Shannon, $p<0.001$ for Simpson, $p=0.001$ for Chao, $p=$ 0.002 for Ace). Furthermore, Mann-Whitney $U$ tests

Table 1 Effects of factors in the Mantel test analysis undertaken on 16 populations where Wolbachia was fixed in the population

\begin{tabular}{llll}
\hline Variation & Effect & $r$ & $P$ \\
\hline Microbial Bray-Curtis dissimilarity & Genetic differentiation $\left(F_{\text {st }}\right)$ & 0.162 & 0.153 \\
& Latitude & -0.106 & 0.678 \\
& Longitude & 0.409 & 0.001 \\
& Annual mean temperature (Bio1) & -0.051 & 0.564 \\
KL divergence & Annual precipitation (Bio12) & 0.306 & 0.029 \\
& Genetic differentiation $\left(F_{\text {st }}\right)$ & 0.163 & 0.089 \\
& Latitude & 0.006 & 0.419 \\
& Longitude & -0.057 & 0.683 \\
& Annual mean temperature (Bio1) & 0.034 \\
\hline
\end{tabular}



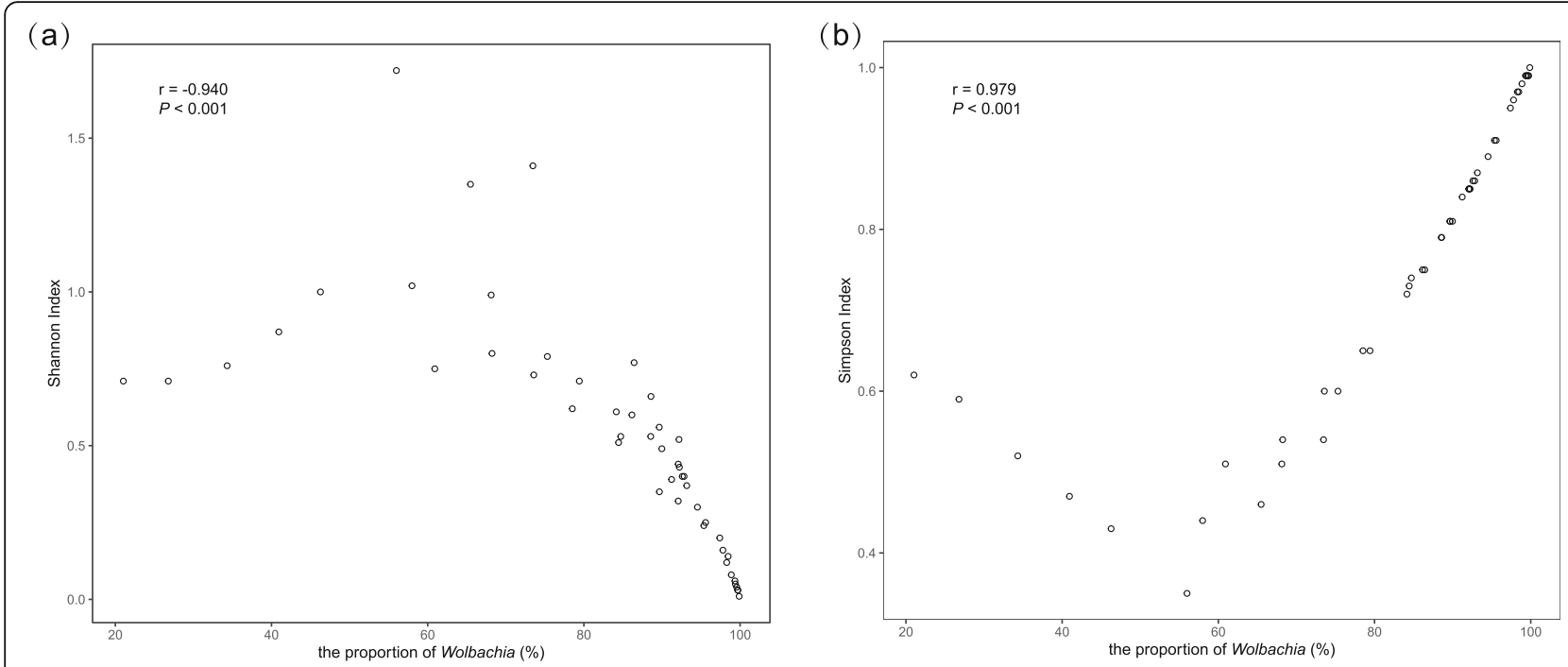

Fig. 5 Relationships between the proportions of Wolbachia and the Shannon indexes (a) or the Simpson indexes (b) of microbial community among the 51 samples by Spearman's rank correlation. $R$ values and $P$ values of each linear regression plots are provided

revealed that the abundances of seven genera that dominated the communities found in the Wolbachia-free adults were very low in the Wolbachia-infected adults (Fig. 7). These results provided further evidence that Wolbachia decreased the relative abundance and diversity in the microbial community of SBPH.

Changes in microbial communities by Wolbachia infection To detect the effect of Wolbachia infection on the structure of the microbial community, we compared microbial taxon abundance between the Wolbachia-infected and Wolbachia-free individuals sampled from the SAP population. To normalize sequencing depth, we haphazardly extracted 1144 reads for each sample (based on the minimum number of reads after removing Wolbachia reads in the Wolbachia-infected samples, Additional file 1: Table S10) for these analyses. Our results showed that the structures of the microbial communities were different between Wolbachia-infected females (after excluding Wolbachia reads) and Wolbachia-uninfected females (Fig. 8a; Additional file 1: Table S11). Both the Shannon and Simpson indexes indicated that the Wolbachia-free group possessed higher microbial diversity than Wolbachia-infected group (excluding Wolbachia reads) (Additional file 1:
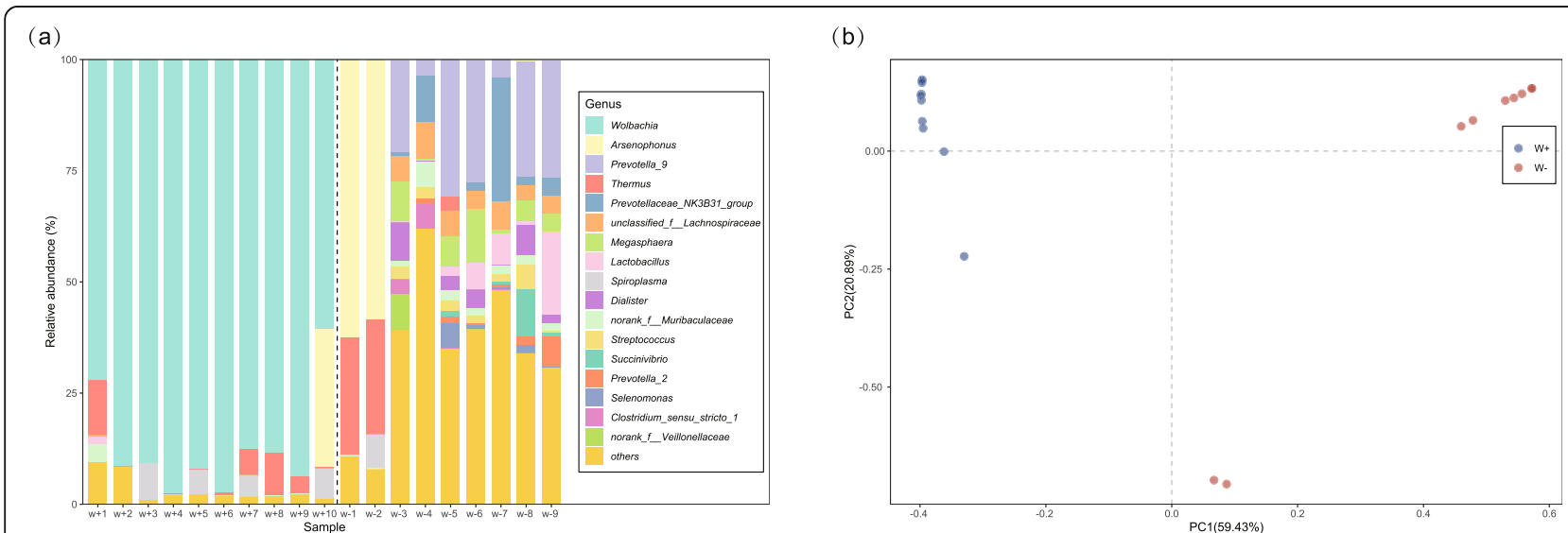

Fig. 6 The abundance and distance of microbial community of SBPH between Wolbachia-infected and Wolbachia-free female adults. a Relative abundance of bacterial 16S rRNA genes at the genus level from 10 Wolbachia-infected female adults and 9 Wolbachia-free female adults in SAP population. Dashed line separates the microbial community abundance of the two groups. Other genera ("others") account for $<5 \%$ of the classified sequences. b Principal coordinate analysis (PCOA) among Wolbachia-infected and Wolbachia-free female adults. PCoA was generated by the Bray-Curtis dissimilarity method 
Table 2 Measures of species richness and evenness of SBPH from 10 Wolbachia-infected females and 9 Wolbachia-free females from the SAP population

\begin{tabular}{lllllll}
\hline Samples & Sobs & Shannon & Simpson & Ace & Chao & Coverage \\
\hline w+1 & 693 & 1.594 & 0.532 & 816.372 & 766.533 & 0.996 \\
$w+2$ & 356 & 0.707 & 0.832 & 434.097 & 409.182 & 0.998 \\
$w+3$ & 76 & 0.378 & 0.829 & 206.037 & 126.647 & 0.999 \\
$w+4$ & 161 & 0.210 & 0.949 & 234.636 & 206.217 & 0.998 \\
$w+5$ & 139 & 0.406 & 0.850 & 188.332 & 178.200 & 0.999 \\
$w+6$ & 132 & 0.206 & 0.943 & 314.430 & 235.542 & 0.998 \\
$w+7$ & 107 & 0.561 & 0.771 & 199.706 & 152.000 & 0.999 \\
$w+8$ & 95 & 0.475 & 0.787 & 209.990 & 159.688 & 0.999 \\
$w+9$ & 96 & 0.337 & 0.877 & 148.918 & 133.625 & 0.999 \\
$w+10$ & 93 & 0.965 & 0.466 & 149.309 & 143.167 & 0.999 \\
$w-1$ & 247 & 1.232 & 0.459 & 342.134 & 312.632 & 0.998 \\
$w-2$ & 402 & 1.490 & 0.412 & 431.463 & 443.143 & 0.998 \\
$w-3$ & 399 & 3.987 & 0.040 & 461.191 & 457.400 & 0.998 \\
$w-4$ & 636 & 5.063 & 0.013 & 689.927 & 684.838 & 0.998 \\
$w-5$ & 528 & 3.990 & 0.052 & 628.688 & 643.000 & 0.997 \\
$w-6$ & 516 & 3.962 & 0.052 & 630.807 & 621.726 & 0.997 \\
$w-7$ & 640 & 4.590 & 0.027 & 709.431 & 722.787 & 0.997 \\
$w-8$ & 534 & 3.915 & 0.052 & 632.849 & 651.018 & 0.997 \\
$w-9$ & 497 & 3.817 & 0.065 & 599.893 & 629.255 & 0.997 \\
\hline & & & & & &
\end{tabular}

Table S12; Welch two-sample $t$ test: $p<0.035$ for Shannon, $p=0.020$ for Simpson). PCoA analysis based on Bray-Curtis dissimilarity (Fig. 8b) also clearly separated the two groups, except for two samples of the Wolbachiauninfected females. Two samples contained very few Wolbachia reads (accounting for $0.04 \%$ of their microbial communities), which might lead to a distorted pattern. However, it appears that even Wolbachia infections at low titers can significantly change the microbial community. In addition to decreasing bacterial diversity, we also found that the Wolbachia infection changed bacterial taxon abundance, with 25 genera significantly increasing and 65 significantly decreasing in Wolbachia-infected individuals (Fig. 9; Additional file 2: Table S13; Table S14; Table S15). Most of these bacteria have widespread distributions in insect tissues, including the gut, ovary, and head. Notably, four genera occurring in high proportions (with log (read percent) >1) in both Wolbachia-infected and Wolbachiafree groups were also significantly different, with Thermus, Spiroplasma, and Ralstonia enriched in the Wolbachia-infected group, in contrast to Prevotella_9 which was enriched in the Wolbachia-uninfected group (Fig. 9). Apart from these changes, Wolbachia infection seems associated with the existence of particular bacterial taxa, with 160 genera specifically existing in relative low abundance in the Wolbachia-infected group (Additional file 2: Table S13).

\section{Wolbachia does not appear to strongly affect immune- related genes of SBPH but affects metabolism genes}

To test if Wolbachia promotes the expression of immune-related genes in SBPH, we compared the transcriptomes of pooled abdomens from Wolbachia-infected and Wolbachia-free females. Of 330 immunerelated genes in SBPH identified by Zhu et al. [45], 306 genes representing 25 gene families were identified (Additional file 2: Table S16). Most of these genes were not differentially expressed (Fig. 10; Additional file 2: Table S16), which suggests that Wolbachia had little or no impact on the immune systems of SBPH. However,

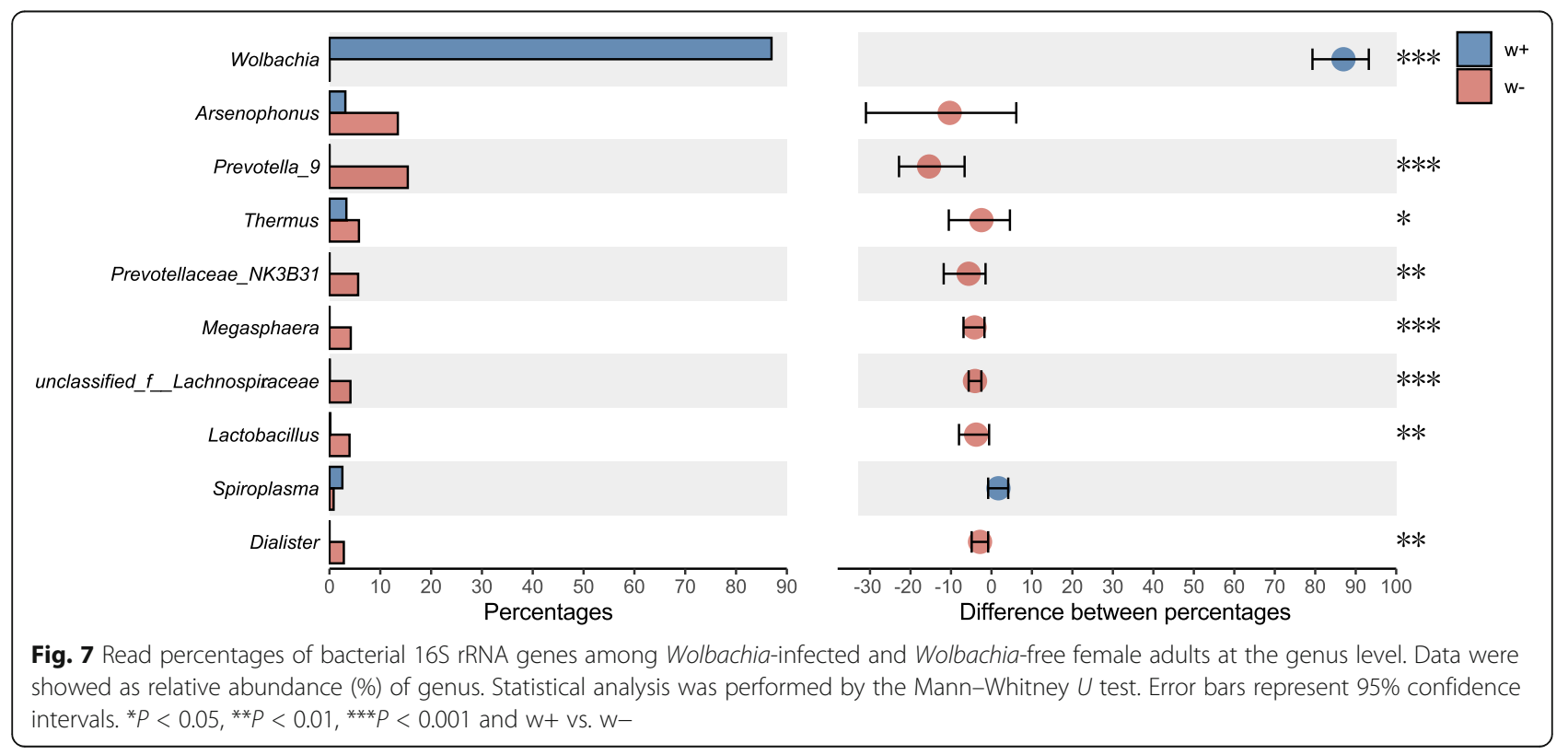




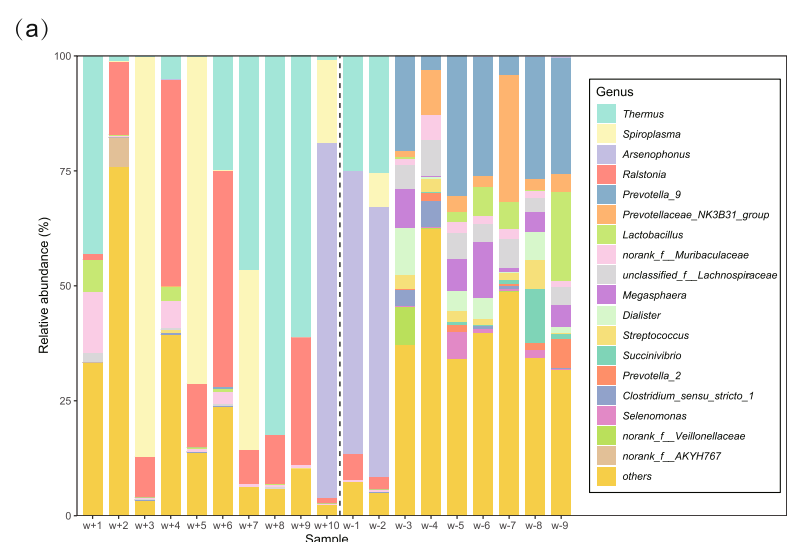

(b)

Fig. 8 Abundance and distance of microbial community of SBPH between Wolbachia-infected (excluding Wolbachia reads) and Wolbachia-free female adults. a Relative abundance of bacterial 16S rRNA genes at the genus level from 10 Wolbachia-infected female (excluding Wolbachia reads) adults and 9 Wolbachia-free female adults in SAP population. The dashed line separates the microbial community abundance of the two groups. Other genera ("others") account for $<5 \%$ of the classified sequences. b Principal coordinate analysis (PCoA) among Wolbachia-infected (excluding Wolbachia reads) and Wolbachia-free female adults. PCoA was generated by the Bray-Curtis dissimilarity method

through an analysis of Kyoto Encyclopedia of Genes and Genomes (KEGG) terms, we found 141 differentially expressed genes in metabolism processes including oxidative phosphorylation-related and glycolysis-related genes (Fig. 10; Additional file 2: Table S17), which suggests that the effect of Wolbachia on microbial community is likely mediated through changing the overall metabolism and physiology of SBPH.

\section{Discussion}

Effects of environmental factors and genetic background on the microbial community of SBPH

Our analyses suggest that, based on Bray-Curtis dissimilarity, longitude and precipitation may impact microbial communities, and these effects appear separate because precipitation did not associate with longitude. To date, any effects of precipitation on insect microbiome have rarely been considered. Our previous study in spider mites found that precipitation can influence the incidence of Spiroplasma [57], a facultative endosymbiont which can manipulate host production. As the $\mathrm{SBPH}$ is polyphagous, any effects of longitude and precipitation may reflect effects of these variables on vegetation and food resources for SBPH, which could alter the physiology and metabolism of SBPH hosts and in turn influence microbial communities. SBPH might acquire some bacteria directly from plant sap, as has been suggested for the cochineal insect Dactylopius [58], and different bacteria present in different environments could contribute to variation in microbial communities. For example, Pantoea was abundant in the MDJ population of $\mathrm{SBPH}$ (Fig. 3a) and is thought to have been acquired from the environment in Ae. albopictus [59]. It is also possible that microbial communities are responding directly to environmental factors rather than being acquired from the environment, and they might even provide a fitness advantage to hosts under certain conditions, although this remains speculative in the absence of experimental data. Future studies should also consider the impacts of variability in climatic variables on microbial communities, whereas we have only considered the average estimates available to us from the tested locations.

Our results based on both Bray-Curtis dissimilarity and KL divergence suggested an association between mtDNA variations and microbial community structure. Previous studies at the macro-evolutionary level have suggested associations between mtDNA variation and microbial communities, but these might reflect geographic isolation that drive speciation rather than genetic backgrounds per se $[20,21]$, whereas our findings from the population level with shallow divergence in the mitochondrial genome [30] provide relatively more direct evidence of an association. Some bacterial groups that are maternally transmitted and living inside cells (like Wolbachia) might be expected to be associated with mtDNA variants which can hitchhike along with spreading endosymbionts [60]. A more recent study in mice found that different mitochondrial genotypes can alter ROS productions, which modulates microbial structure in the host gut [61]. In SBPH, two mitochondrial haplogroups thought to be associated with altered functions exist in natural populations [30], and their impacts on microbial communities could be explored in future work.

\section{The effects of Wolbachia on the microbial community of SBPH}

Maternally inherited Wolbachia endosymbionts are common in insects. They can manipulate host reproduction, facilitating Wolbachia's rapid spread in a host population. 


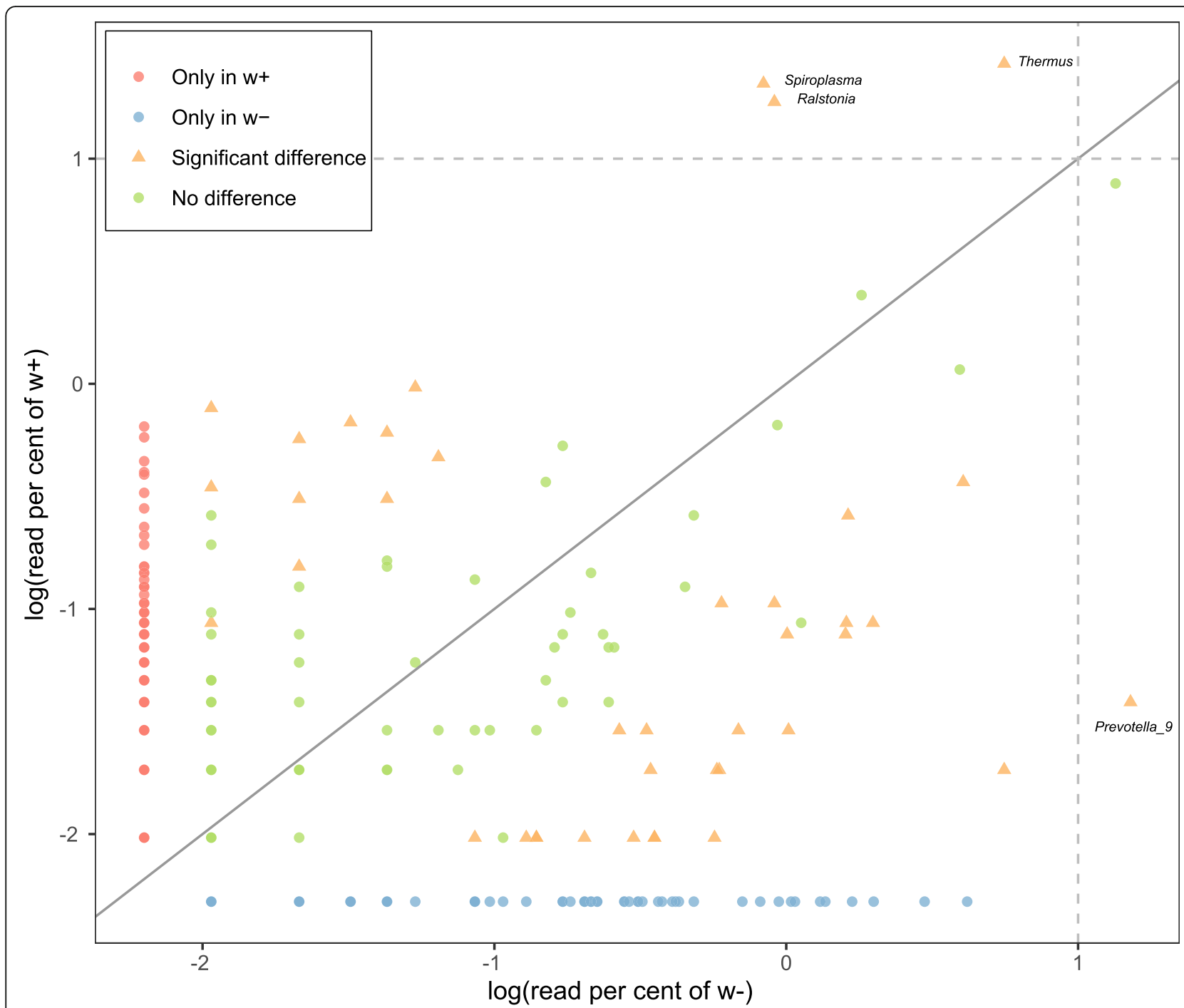

Fig. 9 Common logarithm values of the read percentage of bacterial 16S rRNA genes for each genus across the microbial communities between Wolbachia-infected females (excluding Wolbachia reads) and Wolbachia-uninfected females and comparisons by Mann-Whitney $U$ tests. Significant differences in the genera existing in Wolbachia-infected females (excluding Wolbachia reads) and Wolbachia-uninfected females are indicated by different colors. Proportions where genera in Wolbachia-infected females (excluding Wolbachia reads)/Wolbachia-uninfected females $=1$ is shown as a dotted line

In $\mathrm{SBPH}$, Wolbachia can induce strong cytoplasmic incompatibility (CI), resulting in no offspring when uninfected females mate with infected males [62]. Comparison of the microbial communities of Wolbachia-free and Wolbachia-infected SBPH individuals clearly shows that Wolbachia infection severely decreases the diversity and abundance of bacteria in SBPH. The abundance of the seven other main genera in Wolbachia-infected adults was very low (Fig. 7). A similar phenomenon has been observed in Aedes aegypti, in which a large proportion of bacterial taxa disappeared when Wolbachia was induced by artificial injection [63]. Bacterial diversity was also found to be very low in the gut of Drosophila melanogaster, which is naturally infected with Wolbachia [64].
Significant differences in microbial communities were observed between the Chinese and Japanese populations of SBPH (Fig. 3b). The present results, together with previous studies, suggest that Wolbachia has rapidly spread in SBPH populations during recent decades in both China and Japan. The incidence of Wolbachia has increased from around $90 \%$ in Chinese populations [53] to $100 \%$ [29], and from around $65 \%$ in Japanese populations [54] to more than $90 \%$. The strong CI of Wolbachia and the high migratory ability of SBPH likely contribute to this rapid spread. The spread of Wolbachia seems to have pushed the infection to fixation in the Chinese populations, while the invasion is still ongoing in the Japanese populations. In Japan, spread is most noticeable in high latitude 


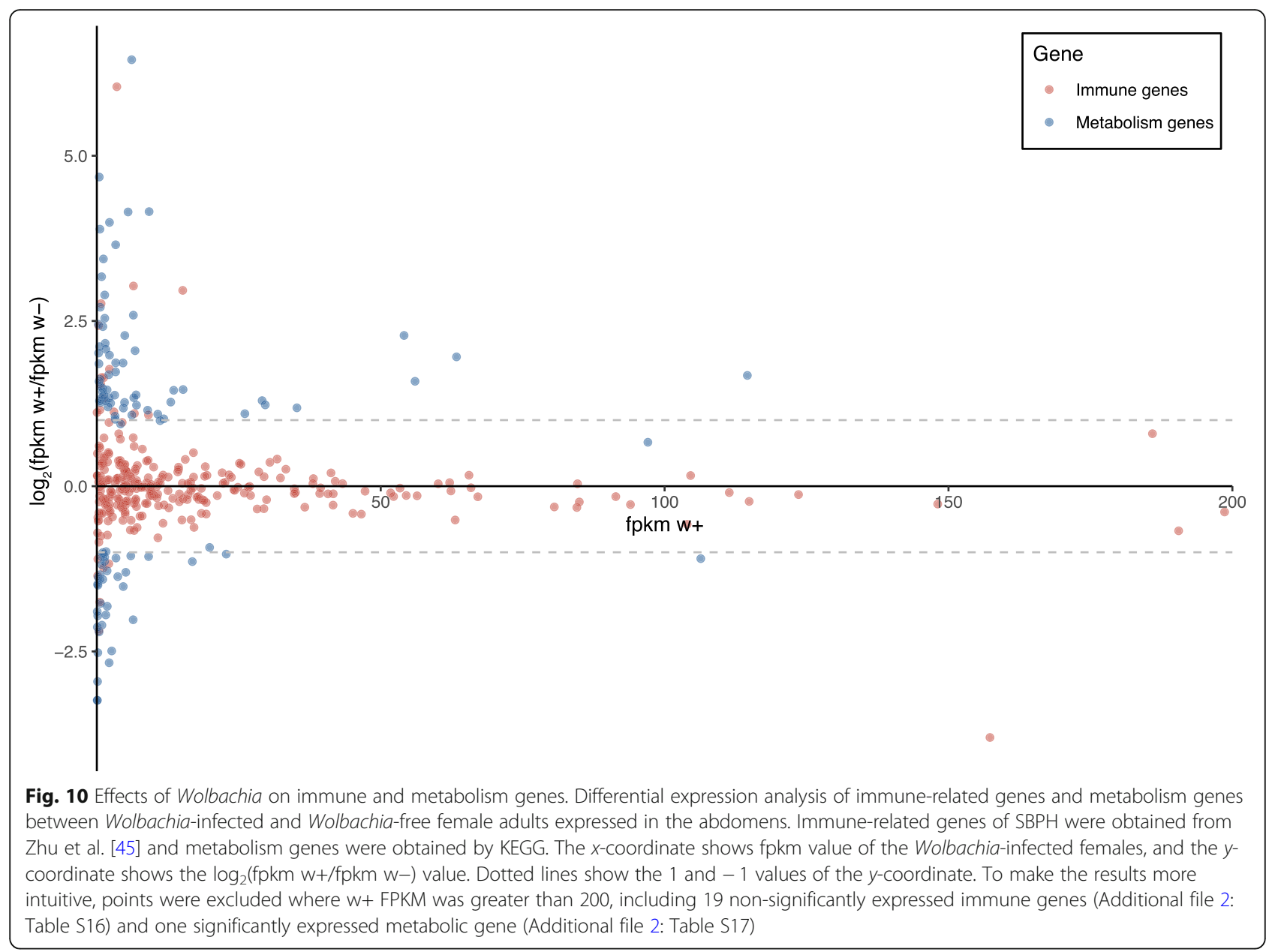

regions where Wolbachia was previously rare. The difference in histories of Wolbachia between China and Japan may be contributing to divergence in their SBPH microbial communities, but this remains to be tested directly, such as through comparisons of the communities when hosts are reared in a common environment.

By removing Wolbachia reads from the Wolbachia-infected females in SAP populations, we further analyzed the effect of Wolbachia on the other bacteria and found that Wolbachia infection changed microbial evenness and other measures of microbial diversity. Three bacteria (Thermus, Spiroplasma, Ralstonia, Fig. 9; Additional file 2: Table S14) were highly enriched in the Wolbachia-infected samples. Vitamin B can be synthesized by Thermus [65], as well as by Wolbachia where it can lead to an increase in host fertility [66]. Thermus associated with Wolbachia may provide an intermediate for the synthesis of vitamin B. In Drosophila neotestacea, Wolbachia can promote the abundance of Spiroplasma [67], pointing to the possibility of direct interactions among microbes. On the other hand, the effect of Wolbachia on Spiroplasma may lead to different tissue tropisms [26] and asymmetrical interactions between the two bacteria where Spiroplasma negatively affects the population of Wolbachia, but Wolbachia does not influence the population of Spiroplasma [26]. In SBPH, Spiroplasma was found to induce late male killing [68] which is predicted to have advantages not only in facilitating maternal transmission, but also in promoting horizontal transmission of Spiroplasma. This is based on the notion that dead males could burst and release Spiroplasma spores into the environment [69]. Whether the bursting of dead males also promotes the spread of other microbes such as Wolbachia in nature is unclear. If so, it could partly contribute to the rapid spread of CI inducing Wolbachia in SBPH populations without decreasing mitochondrial DNA diversity [29]. Ralstonia is a devastating soil-borne plant pathogen and affects growth and development of 200 host species belonging to more than 50 botanical families [70]. For SBPH, we speculated that Ralstonia may have been obtained from food resources, but the function of Ralstonia in insect hosts is unknown. We also note that many bacteria were reduced by Wolbachia infections (Additional file 2: Table S15), and most of them were located in the gut, ovary, and head 
where Wolbachia exist [33]. Wolbachia may interact competitively with many components of the microbial community of SPBH but this remains to be investigated.

The main mechanisms by which Wolbachia are thought to decrease the microbial diversity are immune system modulation and resource competition [63]. Other mechanisms may include Wolbachia-induced changes in ROS, transcription/posttranscription, and $\mathrm{pH}$ [64]. Because no significant difference in the expressions of immunityrelated genes was detected in the transcriptomes of Wolbachia-infected and Wolbachia-free female adults, it appears that immune modulation is not involved in SBPH. The only effect detected in this study was a decrease in the expression of the gene encoding defensin in the Wolbachia-infected females (Additional file 2: Table S16), the opposite of what might be expected. Through KEGG analysis, we showed 141 differentially expression genes involving metabolic processes including oxidative phosphorylation and glycolysis (Additional file 2: Table S17), which may suggest that intracellular localized somatic Wolbachia affect the overall metabolism and physiology of the insect to suppress the diversity/abundance of bacterial populations.

Wolbachia infection-associated immune regulation has been reported in organisms in which Wolbachia was artificially introduced [24, 25, 71], but not in organisms that are naturally infected with Wolbachia $[23,72]$. It seems that immune regulation mediated by Wolbachia is lost with a long history of Wolbachia colonization. The initial colonization of Wolbachia may trigger an immune response in the host, which then changes after long-term co-evolution between Wolbachia and its host. If that is the case, managing insect pests by releasing insects artificially infected with Wolbachia should be undertaken with caution because the "pathogen blocking" efficiency of insect vectors may eventually be lost in nature as Wolbachia and its host co-evolve. We agree with Simhadri et al. [64] who argue that future studies of Wolbachia-associated phenotypes should consider the effects of Wolbachia on the microbial community.

\section{Conclusions}

In this study, by profiling $16 \mathrm{~S}$ rRNA genes using nextgeneration sequencing, we explored the relative contributions of genetic background, ecological factors, and interactions among microbial species on the microbial communities of natural populations of SBPH. Our results suggest that Wolbachia infection has a stronger role in shaping the microbial community than ecological factors and genetic (mtDNA) background. When Wolbachia is introduced into the community, it seems to become the dominant species and decreases microbial diversity. Comparative RNA-Seq analyses between $\mathrm{Wol}$ bachia-infected and -uninfected strains indicate that the Wolbachia do not seem to alter the immune reaction of
SBPH, although Wolbachia affected expression of metabolism genes, suggesting Wolbachia affect the overall metabolism and physiology of the insect to suppress the diversity/abundance of bacterial populations.

\section{Supplementary information}

Supplementary information accompanies this paper at https://doi.org/10. 1186/s40168-020-00878-x.

\begin{abstract}
Additional file 1: Table S1 Summary of collection details. The population code (ID), province, city, county, latitude, longitude, and date of the field collections assessed here are provided. Table S2 Specific primers used in PCR for this study. Table S3 Pairwise $F_{\text {ST }}$ estimates between populations based on a sequence of the mitochondrial $\mathrm{CO}$ gene. Population codes are given in Table S1. Table S4 Annual mean temperatures (Bio1) and the annual mean precipitation (Bio12) of the 17 locations obtained from DIVA-GIS 7.5.0. Table S6 Relative abundance of bacterial 16S rRNA genes at the genus level observed for Chinese, Japanese and all populations. Table S7 Effects of factors in the structural equation model (SEM) analysis undertaken on 16 populations where Wolbachia was fixed in the population. Table S10 After Wolbachia was excluded from the Wolbachia-infected adults, the composition of all samples from SAP populations. Table S11 Relative abundance of bacterial 165 rRNA genes at the genus level observed for Wolbachia-infected females (after removal of Wolbachia reads), Wolbachia-uninfected females and all samples. Table S12 Measures of species richness and evenness of SBPH from 10 Wolbachia-infected females (excluding Wolbachia reads) and 9 Wolbachia-free females from the SAP population.

Additional file 2: Table S5 Abundance of OTUs among the 48 samples. Table S8 Abundance of OTUs between the Wolbachia-infected and Wolbachia-free females in SAP populations. Table S9 Read proportions of bacterial 165 rRNA genes among Wolbachia-infected females and Wolbachia-free females at the genus level by Mann-Whitney $\mathrm{U}$ tests. Table S13 Read proportions of bacterial 16S rRNA genes for Wol bachia-infected females (excluding Wolbachia reads) at the genus level by Mann-Whitney $U$ tests. Table S14 Significantly increased read proportions of bacterial $16 \mathrm{~S}$ rRNA genes and the tissue in which their corresponding bacteria were distributed for Wolbachia-infected females (excluding Wolbachia reads) at the genus level by Mann-Whitney $U$ tests. The tissue distribution of bacteria was obtained from Zhang et al. [33]. Table S15 Significantly reduced read proportions of bacterial 16S rRNA genes and the tissue in which their corresponding bacteria were distributed for Wolbachia-infected females (excluding Wolbachia reads) at the genus level by Mann-Whitney $U$ tests. The tissue distribution of bacteria was obtained from Zhang et al. [33]. Table S16 Expression differences of immune genes between Wolbachia-infected and Wolbachia-cured female adults expressed in abdomens. Table S17 Expression differences of metabolism genes between Wolbachia-infected and Wolbachia-cured female adults expressed in abdomens.
\end{abstract}

\section{Acknowledgements}

We are very grateful to Drs. Takashino K, Sano M, Matsukura M, Fujii T, Sanada S, and Izumi Y (NARO Kyushu Okinawa Agricultural Research Center, Japan) for help with collection of Japanese populations; Lei Chen for help with collection of Chinese populations; Dr. Hai-Jian Huang of Ningbo University, Zhejiang Province, China, for reviewing an early draft of the manuscript and for providing suggestions; and Dr. Jie Li of Hokkaido University, Hokkaido, Japan, for analyzing probabilistic features of OTU distribution. We also thank Professor William Sullivan, Department of MCD Biology, University of California at Santa Cruz, USA, for his valuable comments and suggestions on the manuscript.

\section{Authors' contributions}

JTS, XYH, and XZD designed the research. JTS, XZD, and MK collected samples. XZD, LTW, and YG performed the research. XZD and XHS conducted the data analyses. $Y X Z, A A H$, and XLB advised on interpretations. $X Z D$, JTS, AAH, and XYH wrote the paper. The authors read and approved the final manuscript. 


\section{Funding}

This work was supported by the National Natural Science Foundation of China (No. 31972264, 31672035 and 31871976). AAH was supported by an NHMRC Research Fellowship while contributing to this paper. The funders had no role in study design, data collection and analysis, decision to publish, or preparation of the manuscript.

\section{Availability of data and materials}

The raw reads of $16 \mathrm{~S}$ rRNA sequencing have been deposited in the NCBI Sequence Read Archive (SRA) database (accession number SRP238740). The raw reads of transcriptomes have been deposited in the NCBI SRA database (accession number SRP195568).

\section{Ethics approval and consent to participate}

Not applicable.

\section{Consent for publication}

Not applicable.

\section{Competing interests}

The authors declare that they have no competing interests.

\section{Author details}

${ }^{1}$ Department of Entomology, Nanjing Agricultural University, Nanjing 210095, Jiangsu, China. ${ }^{2}$ NARO Kyushu Okinawa Agricultural Research Center, 2421 Suya, Koshi, Kumamoto 861-1192, Japan. ${ }^{3}$ School of BioSciences, Bio21 Institute, The University of Melbourne, Melbourne, Victoria 3010, Australia.

\section{Received: 3 May 2020 Accepted: 1 June 2020}

\section{Published online: 02 July 2020}

\section{References}

1. Moran NA, McCutcheon JP, Nakabachi A. Genomics and evolution of heritable bacterial symbionts. Annu Rev Genet. 2008;42:165-90.

2. Moya A, Peretó J, Gil R, Latorre A. Learning how to live together: genomic insights into prokaryote-animal symbioses. Nat Rev Genet. 2008;9:218.

3. Feldhaar H. Bacterial symbionts as mediators of ecologically important traits of insect hosts. Ecol Entomol. 2011;36:533-43.

4. Coon KL, Vogel KJ, Brown MR, Strand MR. Mosquitoes toes rely on their gut microbiota for development. Mol Ecol. 2014;23:2727-39.

5. Duron O, Bouchon D, Boutin S, Bellamy L, Zhou L, Engelstädter J, et al. The diversity of reproductive parasites among arthropods: Wolbachia do not walk alone. BMC Biol. 2008;6:27.

6. Brownlie JC, Johnson KN. Symbiont-mediated protection in insect hosts. Trends Microbiol. 2009;17:348-54.

7. Kriesner P, Conner WR, Weeks AR, Turelli M, Hoffmann AA. Persistence of Wolbachia infection frequency cline in Drosophila melanogaster and the possible role of reproductive dormancy. Evolution. 2016;70:979-97.

8. Douglas AE. Nutritional interactions in insect-microbial symbioses: aphids and their symbiotic bacteria Buchnera. Annu Rev Entomol. 1998;43:17-43.

9. Akman GE, Douglas AE. Symbiotic bacteria enable insect to use a nutritionally inadequate diet. Proc R Soc B. 2009;276:987-91.

10. Douglas AE. Lessons from studying insect symbioses. Cell Host Microbe. 2011;10:359-67.

11. Raymann K, Shaffer Z, Moran NA. Antibiotic exposure perturbs the gut microbiota and elevates mortality in honeybees. PLoS Biol. 2017;15:e2001861.

12. Gaio AO, Gusmão DS, Santos AV, Berbert-Molina MA, Pimenta PFP, Lemos FJA. Contribution of midgut bacteria to blood digestion and egg production in Aedes aegypti (diptera: Culicidae). Parasites Vectors. 2011;4:105.

13. Gendrin M, Rodgers FH, Yerbanga RS, Ouedraogo JB, Basanez MG, Cohuet A, et al. Antibiotics iningested human blood affect the mosquito microbiota and capacityto transmit malaria. Nat Commun. 2015;6:5921.

14. Santo DJW, Kaufman MG, Klug MJ, Holben WE, Harris D, Tiedje JM. Influence of diet on the structure and function of the bacterial hindgut community of crickets. Mol Ecol. 1998;7:761-7.

15. Schmitt-Wagner D, Friedrich MW, Wagner B, Brune A. Axial dynamics, stability, and interspecies similarity of bacterial community structure in the highly compartmentalized gut of soil-feeding termites (Cubitermes spp.). Appl Environ Microbiol. 2003;69:6018-24.

16. Hongoh $Y$, Deevong $P$, Inoue T, Moriya S, Trakulnaleamsai S, Ohkuma M, et al. Intra- and interspecific comparisons of bacterial diversity and community structure support coevolution of gut microbiota and termite host. Appl Environ Microbiol. 2005;71:6590-9.

17. Mohr Kl, Tebbe CC. Diversity and phylotype consistency of bacteria in the guts of three bee species (Apoidea) at an oilseed rape field. Environ Microbiol. 2006;8:258-72.

18. Behar A, Yuval B, Jurkevitch E. Community structure of the mediterranean fruit fly microbiota: seasonal and spatial sources of variation. Isr J Ecol Evol. 2008;54:181-91.

19. Suzuki TA, Phifer-Rixey M, Mack KL, Sheehan MJ, Lin DN, Bi K, et al. Host genetic determinants of the gut microbiota of wild mice. Mol Ecol. 2019;28:3197-207.

20. Colman DR, Toolson EC, Takacs-Vesbach CD. Do diet and taxonomy influence insect gut bacterial communities? Mol Ecol. 2012;21:5124-37.

21. Sanders JG, Powell S, Kronauer DJ, Vasconcelos HL, Frederickson ME, Pierce NE. Stability and phylogenetic correlation in gut microbiota: lessons from ants and apes. Mol Ecol. 2014;23:1268-83.

22. Brinker P, Fontaine MC, Beukeboom LW, Falcao SJ. Host, symbionts, and the microbiome: the missing tripartite interaction. Trends Microbiol. 2019;27:480-8

23. Rancès $E, Y e$ YH, Woolfit M, McGraw EA, O'Neill SL. The relative importance of innate immune priming in Wolbachia-mediated dengue interference. PLoS Pathog. 2012:8:e1002.

24. Moreira LA, Iturbe-Ormaetxe I, Jeffery JA, Lu GJ, Pyke AT, Hedges LM, et al. A Wolbachia symbiont in Aedes aegypti limits infection with dengue, chikungunya, and plasmodium. Cell. 2009;139:1268-78.

25. Bian G, Xu Y, Lu P, Xie Y, Xi ZY. The endosymbiotic bacterium Wolbachia induces resistance to dengue virus in Aedes aegypti. PLoS Pathog. 2010;6:e1000833.

26. Goto S, Anbutsu H, Fukatsu T. Asymmetrical interactions between Wolbachia and Spiroplasma endosymbionts coexisting in the same insect host. Appl Environ Microbiol. 2006;72:4805-10.

27. Hughes $\mathrm{GL}$, Dodson BL, Johnson RM. Native microbiome impedes vertical transmission of Wolbachia in Anopheles mosquitoes. Proc Natl Acad Sci U S A. 2014;111:12498-503.

28. Otuka A, Matsumura M, Sanada-Morimura S, Takeuchi H, Watanabe T, Ohtsu $\mathrm{R}$, et al. The 2008 overseas mass migration of the small brown planthopper, Laodelphax striatellus, and subsequent outbreak of rice stripe disease in western Japan. Appl Entomol Zool. 2010;45:259-66.

29. Sun JT, Wang MM, Zhang YK, Chapuis MP, Jiang XY, Hu G, et al. Evidence for high dispersal ability and Mito-nuclear discordance in the small brown planthopper, Laodelphax striatellus. Sci Rep. 2015;5:8045.

30. Sun JT, Duan XZ, Hoffmann AA, Liu Y, Garvin MR, Chen L, et al. Mitochondrial variation in small brown planthoppers linked to multiple traits and probably reflecting a complex evolutionary trajectory. Mol Ecol. 2019;28:3306-23.

31. Yang XQ, Wang ZL, Wang TZ, Yu XP. Analysis of the bacterial community structure and diversity in the small brown planthopper, Laodelphax striatellus (Hemiptera: Delphacidae) by 165 rRNA high-throughput sequencing. Acta Ent. Sin. 2018;61:200-8.

32. Li S, Zhou C, Chen G, Zhou Y. Bacterial microbiota in small brown planthopper populations with different rice viruses. J Basic Microbi. 2017;57:590-6.

33. Zhang X, Li TP, Zhou CY, Zhao DS, Zhu YX, Bing XL, et al. Antibiotic exposure perturbs the bacterial community in the small brown planthopper Laodelphax striatellus. Insect Sci. 2019;00:1-13.

34. Chandler JA, Lang JM, Bhatnagar S, Eisen JA, Kopp A. Bacterial communities of diverse Drosophila species: ecological context of a host-microbe model system. PLoS Genet. 2011;7:e1002272.

35. Keenan SW, Engel AS, Elsey RM. The alligator gut microbiome and implications for archosaur symbioses. Sci Rep. 2013;3:2877.

36. Clayton JB, Vangay P, Huang H, Ward T, Hillmann BM, Al-Ghalith GA, et al. Captivity humanizes the primate microbiome. Proc Natl Acad Sci U S A. 2016:113:10376-81.

37. Illumina. 16S Metagenomic Sequencing Library Preparation Guide. 2013. https://support.illumina.com/downloads/16s_metagenomic_sequencing_ library_preparation.html.

38. Magoc T, Salzberg SL. FLASH: fast length adjustment of short reads to improve genome assemblies. Bioinformatics. 2011;27:2957-63.

39. Edgar RC. UPARSE: highly accurate OTU sequences from microbial amplicon reads. Nat Methods. 2013;10:996-8.

40. DeSantis TZ, Hugenholtz P, Larsen N, Rojas M, Brodie EL, Keller K, et al. Greengenes, a chimera-checked 16S rRNA gene database and workbench compatible with ARB. Appl Environ Microbiol. 2006;72:5069-72.

41. Zhao DX, Zhang YK, Chen H, Hong XY. Effects of transinfection of Wolbachia from Laodelphax striatellus (Hemiptera: Delphacidae) on 
reproductive fitness and adult longevity of Tetranychus phaselus (Acari: Tetranychidae). Acta Ent Sin. 2014:57:25-35.

42. Guo Y, Hoffmann AA, XU XQ, Zhang X, Huang HJ, Ju JF, et al. Wolbachiainduced apoptosis associated with increased fecundity in Laodelphax striatellus (Hemiptera: Delphacidae). Insect Mol Biol. 2018;27:796-807.

43. Ju JF, Hoffmann AA, Zhang YK, Duan XZ, Guo Y, Gong JT, et al. Wolbachiainduced loss of male fertility is likely related to branch chain amino acid biosynthesis and iLvE in Laodelphax striatellus. Insect Biochem Mol Biol. 2017;85:11-20

44. Li B, Dewey CN. RSEM: accurate transcript quantification from RNA-Seq data with or without a reference genome. BMC Bioinf. 2011;12:323.

45. Zhu J, Jiang F, Wang X, Yang PC, Bao YY, Zhao W, et al. Genome sequence of the small brown planthopper. Laodelphax striatellus Gigascience. 2017;6:1-12.

46. Altschul SF, Gish W, Miller W, Myers EW, Lipman DJ. Basic local alignment search tool. J Mol Bio. 1990;215:403-10.

47. Caporaso JG, Kuczynski J, Stombaugh J, Bittinger K, Bushman FD, Costello EK, et al. QIIME allows analysis of high-throughput community sequencing data. Nat Methods. 2010;7:335-6.

48. Excoffier $L$, Lischer HE. Arlequin suite ver 3.5: a new series of programs to perform population genetics analyses under Linux and windows. Mol. Ecol. Resour. 2010;10:564-7.

49. Robert JH, Mariana Cruz, Edwin Roja, Guarino L. Title of subordinate document. In: DIVA-GIS Version 1.4 a geographic information system for the management and analysis of genetic resources data. Manual. International Potato Center. 1999. http://www.diva-gis.org/docs/pgr127_15-19.pdf. Accessed Jan 2001.

50. Hayduk LA. Structural equation modeling with LISREL: essentials and advances. Baltimore: The Johns Hopkins University Press; 1987.

51. R Development Core Team. R: A language and environment for statistical computing. Vienna: R Foundation for Statistical Computing; 2015. http:// www.r-project.org. Accessed 10 Feb 2015.

52. Li J, Convertino M. Optimal microbiome networks: macroecology and criticality. Entropy. 2019;21:506.

53. Zhang K, Han X, Hong XY. Various infection status and molecular evidence for horizontal transmission and recombination of Wolbachia and Cardinium among rice planthoppers and related species. Insect Sci. 2013;20:329-44.

54. Hoshizaki S, Shimada T. PCR-based detection of Wolbachia, cytoplasmic incompatibility microorganisms, infected in natural populations of Laodelphax striatellus (Hornoptera: Delphacidae) in Central Japan: has the distribution of Wolbachia spread recently? Insect Mol Biol. 1995;4:237-43.

55. Shannon CE. A mathematical theory of communication. Bell Syst Tech J. 1948;27:379-423.

56. Simpson EH. Measurement of diversity. Nature. 1949;163:688.

57. Zhu YX, Song YL, Zhang YK, Hoffmann AA, Zhou JC, Sun JT, et al. Incidence of facultative bacterial endosymbionts in spider mites associated with local environments and host plants. Appl Environ Microbiol. 2018;84:e02546-17.

58. Ramirez-Puebla ST, Rosenblueth M, Chavez-Moreno CK, de Lyra MCCP, Tecante A, Martinez-Romero E. Molecular phylogeny of the genus Dactylopius (Hemiptera: Dactylopiidae) and identification of the symbiotic bacteria. Environ Entomol. 2010;39:1178-83.

59. Moro CV, Tran FH, Raharimalala FN, Ravelonandro P, Mavingui P. Diversity of culturable bacteria including Pantoea in wild mosquito Aedes albopictus. BMC Microbiol. 2013;13:70.

60. Hale LR, Hoffmann AA. Mitochondrial DNA polymorphism and cytoplasmic incompatibility in natural populations of Drosophila simulans. Evolution. 1990;44:1383-6.

61. Yardeni $T$, Tanes CE, Bittinger K, Mattei LM, Schaefer PM, Singh LN, et al. Host mitochondria influence gut microbiome diversity: a role for ROS. Sci Signal. 2019;12:eaaw3159.

62. Noda H, Koizumi Y, Zhang Q, Deng KJ. Infection density of Wolbachia and incompatibility level in two planthopper species, Laodelphax striatellus and Sogatella furcifera. Mol Biol. 2001;31:727-37.

63. Audsley MD, Seleznev A, Joubert DA, Woolfit M, O'Neill SL, McGraw EA. Wolbachia infection alters the relative abundance of resident bacteria in adult Aedes aegypti mosquitoes, but not larvae. Mol Ecol. 2017;27:297-309.

64. Simhadri RK, Fast EM, Guo R, Schultz MJ, Vaisman N, Ortiz L, Frydman HM et al.. The gut commensal microbiome of Drosophila melanogaster is modified by the endosymbiont Wolbachia mSphere. 2017;2:e00287-17.

65. Rehse PH, Kitao T, Tahirov TH. Structure of a closed-form uroporphyninogen-lll Cmethyltransferase from Thermus thermophilus. Acta Crystallogr, sect. D. 2005;61:913-9.
66. Ju JF, Bing XL, Zhao DS, Guo Y, Xi ZY, Hoffmann AA, et al. Wolbachia supplement biotin and riboflavin to enhance reproduction in planthoppers. ISME J. 2019;14:676-87.

67. Fromont C, Adair KL, Douglas AE. Correlation and causation between the microbiome, Wolbachia and host functional traits in natural populations of drosophilid flies. Mol Ecol. 2019;28:1826-41.

68. Sanada-Morimura S, Matsumura M, Noda H. Male killing caused by a Spiroplasma symbiont in the small brown planthopper. Laodelphax striatellus J Hered. 2013;104:821-9.

69. Hurst GDD, Majerus MEN. Why do maternally inherited microorganisms kill males. Heredity. 1993;71:81-95.

70. Salanoubat M, Genin S, Artiguenave F, Gouzy J, Mangenot S, Arlat M, et al. Genome sequence of the plant pathogen Ralstonia solanacearum. Nature. 2002:415:497-502.

71. Kambris Z, Cook PE, Phuc HK, Sinkins SP. Immune activation by lifeshortening Wolbachia and reduced filarial competence in mosquitoes. Science. 2009:326:134-6.

72. Shi M, White VL, Schlub T, Eden JS, Hoffmann AA, Holmes EC. No detectable effect of Wolbachia wMel on the prevalence and abundance of the RNA virome of Drosophila melanogaster. Proc R Soc B. 2018;285:20181165.

\section{Publisher's Note}

Springer Nature remains neutral with regard to jurisdictional claims in published maps and institutional affiliations.

\section{Ready to submit your research? Choose BMC and benefit from:}

- fast, convenient online submission

- thorough peer review by experienced researchers in your field

- rapid publication on acceptance

- support for research data, including large and complex data types

- gold Open Access which fosters wider collaboration and increased citations

- maximum visibility for your research: over $100 \mathrm{M}$ website views per year

At $\mathrm{BMC}$, research is always in progress.

Learn more biomedcentral.com/submissions 